\title{
L'histoire de la pensée juridique : historiographie, actualité et enjeux
}

\section{Géraldine Cazals et Nader Hakim}

(1) https://publications-prairial.fr/cliothemis/index.php?id=676

DOI : 10.35562/cliothemis.676

\section{Référence électronique}

Géraldine Cazals et Nader Hakim, « L'histoire de la pensée juridique :

historiographie, actualité et enjeux ", Clio@Themis [En ligne], 14 | 2018, mis en ligne le 30 mars 2021, consulté le 24 avril 2021. URL : https://publicationsprairial.fr/cliothemis/index.php?id=676

\section{Droits d'auteur}

CC BY-NC-SA 


\title{
L'histoire de la pensée juridique : historiographie, actualité et enjeux
}

\author{
Géraldine Cazals et Nader Hakim
}

\section{TEXTE}

1 Entre toutes les tendances actuelles de l'histoire du droit en France, l'émergence de l'histoire de la pensée juridique en tant que champ de recherche de premier plan constitue un phénomène notable. Depuis les années 1950, comme l'attestent notamment les travaux de Michel Villey, l'histoire de la pensée juridique s'est considérablement développée en France, en lien avec l'histoire de la philosophie du droit. Dans les années 1980, à l'invitation des juristes privatistes et publicistes, les études s'attachant au premier chef à l'analyse des auteurs, de leurs idées, constructions et doctrines se sont multipliées, trouvant à s'exprimer dans plusieurs revues juridiques en grande partie spécialisées telles la revue Droits. Revue française de théorie juridique (depuis 1985) ou les Annales d'histoire des Facultés de droit devenues Revue d'histoire des facultés de droit et de la science juridique (depuis 1985). Sensibles à ce mouvement, les historiens du droit ont récemment investi massivement ce champ de la recherche, en s'attelant notamment à mettre en avant l'histoire de la pensée juridique contemporaine, en lien avec certains champs d'études juridiques particuliers (doctrine civiliste, administrativiste ou travailliste par exemple), et avec, en toile de fond, certains questionnements méthodologiques développés dans les années 1970. De ce dynamisme témoigne aujourd'hui le développement d'enseignements spécifiques consacrés par les historiens du droit à l'histoire de la pensée juridique. Si traditionnellement, en effet, l'histoire de la pensée juridique était présente dans les cours de philosophie, de culture juridique ou de théorie comme dans les cours d'histoire du droit privé ou public, depuis une petite dizaine d'années, à l'initiative d'historiens, de privatistes et de publicistes, des enseignements de spécialités sont apparus (pas moins de dix-huit cours dans quinze établissements d'enseignement supérieur), auxquels il convient d'ajouter le Master d'histoire de la pensée juridique issu de la collaboration entre les universités de Paris Panthéon-Sorbonne et de Paris Descartes. 
2 Malgré ces avancées, force est de constater que l'histoire de la pensée juridique reste encore en très grande partie à défricher, notamment dans ses aspects historiographiques. Parmi les historiens du droit (pour le moins en France), l'intérêt de l'historiographie en effet ne fait pas toujours l'unanimité. Il y a là, pour certains, des réflexions sans intérêt, pour d'autres, des perspectives relevant d'une sphère qui devrait rester personnelle à l'historien, d'un processus psychique dont la mise à jour relèverait d'une sorte de psychanalyse ne devant en aucun cas faire l'objet de débats « scientifiques ». Dans un tel contexte, l'effort opéré par une partie des historiens du droit français pour développer les débats historiographiques au sein de notre discipline, voire au cœur même de lieux institutionnels hautement symboliques de cette dernière, doit être salué. Le colloque organisé par la Société d'histoire du droit à Toulouse, à l'heureuse initiative de Jacques Poumarède (en 2005), comme le volume composé sous l'égide de l'Association des historiens des facultés de droit dirigé par Jacques Krynen et Bernard d'Alteroche, L'histoire du droit en France. Nouvelles tendances, nouveaux territoires (2014), sont à cet égard emblématiques. Pour autant, comme ce dernier volume en témoigne, beaucoup restent à faire. Non seulement parce que dans le cadre du travail voulu par l'Association des historiens des facultés de droit il ne pouvait être question que de synthèses rapides, mais également parce que l'exercice demandé portait sur une histoire faite en France par les historiens du droit en poste dans les facultés de droit, et qu'un travail historiographique digne de ce nom se doit naturellement de s'inscrire dans une réflexion plus vaste, ayant également pour horizon d'autres histoires faite aussi hors des frontières par un certain nombre de collègues qu'intéresse aussi l'histoire du droit en France et en Europe, voire un travail plus vaste encore.

Il n'est qu'à observer l'extraordinaire vitalité des études historiographiques conduites par nos collègues des facultés de lettres pour mesurer l'intérêt que peut présenter en histoire du droit, et en histoire de la pensée juridique tout particulièrement, le développement d'une approche historiographique plus fouillée. Depuis au moins trente ans (mais en réalité davantage) l'historiographie a donné lieu chez les historiens des lettres à des travaux pléthoriques, à tel point que qu'il y a là désormais un chantier majeur de l'histoire, lequel a contribué à « redéfinir l'identité de la discipline dans son rapport privilégié au 
temps ${ }^{1}$. Deux perspectives essentielles ont été envisagées. La première s'est attachée à prendre en compte l'historicité des sociétés étudiées par l'historien ; la seconde a consisté à prendre pleine conscience de l'historicité propre au travail de l'historien. Le tout a donné lieu à une intense période de réflexivité pour la discipline. Et si cette réflexivité a peut-être contribué dans les années 1980 à accroître le sentiment d'une crise de l'histoire, force est de constater qu'elle a permis à cette dernière de se départir de certains schémas interprétatifs par trop conditionnés par l'histoire politique et intellectuelle du $\mathrm{xx}^{\mathrm{e}}$ siècle, et de renouveler en profondeur les perspectives de la discipline, questionnant à nouveau le rôle social incombant à l'historien dans la société contemporaine ${ }^{2}$.

4 C'est à une telle rénovation de notre approche de l'histoire de la pensée juridique que le présent volume espère contribuer. Considérant l'importance des différentes orientations qui peuvent être celles de l'histoire de la pensée juridique, il ne saurait y contribuer que de manière partielle, voire pointilliste. Nous avons souhaité qu'il le fasse de deux manières : en faisant appel aux témoignages de certains des acteurs majeurs du développement de l'histoire de la pensée juridique à l'échelle internationale ${ }^{3}$, comme en laissant place ensuite à des articles de fond sur le renouvellement historiographique actuel concernant les différentes époques concernées par l'histoire de la pensée juridique.

Au-delà de la diversité des problématiques envisagées et des moments considérés, un certain nombre de points nodaux nous semblent devoir ressortir de l'ensemble des contributions ici présentées.

Ce qui découle en premier lieu des témoignages comme des études de fonds ici présentés, c'est que l'histoire de la pensée juridique, dans son développement comme dans les attentes qu'elle fait naître, tend à dépasser une approche technicienne du droit liée à une vision étroitement positiviste et réductrice des phénomènes juridiques. Loin d'être purement théorique, cet enjeu s'avère primordial, tout en restant indissociable d'engagements personnels forts. C'est qu'il s'agit d'en finir avec une conception du droit ne relevant que d'une approche purement formelle, abstraite et identifiée sans esprit critique à une science pure, une « reine Rechtslehre » mal comprise (P. Grossi). 
L'enjeu est ainsi d'intégrer des facteurs tout à la fois économiques, politiques, sociaux et individuels dans une étude du droit qui ne regarde plus ce dernier comme un objet isolé (M. Stolleis). Tout en permettant de répondre à l'ennui éprouvé par certains étudiants sur les bancs d'universités restées par trop attachées à des enseignements techniques, il s'agit aussi de réagir à une perte de sens, quitte à s'exposer soi-même : l'histoire de la pensée juridique pouvant apparaitre comme un véritable «sport de combat » (C. Jamin). Démontrant l'irréductibilité du droit à des institutions ou des techniques, donnant à voir la complexité des phénomènes juridiques et renouant les fils distendus entre droit et société, l'histoire de la pensée juridique continue de se heurter ce faisant à une approche dogmatique et utilitariste du droit. Contribuant à dénoncer les incapacités actuelles à penser l'ordre juridique contemporain et l'épuisement des modèles politiques et juridiques, elle est invitée à préparer les nouvelles configurations juridiques de demain, à « sonder tout ce processus, pour le corriger et l'orienter ; et d'abord le maîtriser analytiquement » (A. Schiavone). Peu importe dès lors, au-delà de spécificités qui peuvent apparaître comme propres à différentes approches, qu'elle soit faite en France par les historiens appartenant statutairement aux facultés de droit, rattachés à la Section 03 du Conseil national des universités, ou qu'elle soit écrite par d'autres spécialistes tels les administrativistes et civilistes relevant des sections 01 ou 02 du Conseil national des Universités (F. Melleray).

Attachés à dépasser une histoire désincarnée des doctrines, les travaux contemporains portant sur l'histoire de la pensée juridique s'efforcent de comprendre au plus près ce que pouvait être la pensée juridique propre aux sociétés étudiées. Leur attention croissante s'est portée sur la nécessité de prendre en compte plusieurs phénomènes parmi lesquels quatre sont apparus ici de manières récurrentes : 1 . la textualité du droit ; 2. la contextualité de la pensée juridique ; 3. les perspectives nationales voire nationalistes qui la conditionnent souvent ; 4. l'utilité des analyses matérielles et bibliométriques permettant d'échapper à certaines considérations teintées d'anachronismes.

8 La textualité $d u$ droit. Faisant écho aux travaux contemporains associant «droit et littérature », les études portant sur l'histoire de la pensée juridique reflètent la nécessité d'ouvrir les yeux sur le fait d'évidence selon lequel bien souvent le droit nous est transmis par 
des textes. L'attention portée à la textualité du droit revient ainsi à ne pas faire l'impasse sur cette dimension inéluctable comme à réinscrire le droit dans le cadre des pratiques discursives de leur temps. À différentes époques répondent en effet différentes figures pratiques, argumentatives ou rhétoriques. Ainsi en est-il dans les œuvres des juristes romains, dont il convient d'approcher le contenu technique avec « la pleine conscience qu'il nous est transmis par l'intermédiaire de l'écriture » (D. Mantovani). Ainsi en est-il également à la Renaissance, alors qu'une extraordinaire créativité pousse les juristes à inventer de nouvelles formes et genres d'expression du droit (G. Cazals). Il en va de même à l'Époque contemporaine, où, le texte juridique apparaît encore comme un système sémiotique, qu'il convient de disséquer pour en analyser les ressorts internes et les liens externes (N. Hakim et A. Monti).

La contextualité de la pensée juridique. Historiciser l'histoire de la pensée juridique, la resituer en son temps en lien avec des perspectives culturelles et intellectuelles plus vastes, par rapport aux objets textuels contemporains, paraît également une nécessité que l'on cherche à étudier qu'il s'agisse à nouveau de la pensée des juristes romains (D. Mantovani), de celle des auteurs de la Renaissance (G. Cazals) ou des œuvres contemporaines (N. Hakim et A. Monti, P.-N. Barenot, P. Decoux). Seul un tel travail permet d'identifier les règles rhétoriques et d'énonciation, les présupposés intellectuels et esthétiques des temps considérés, et de réduire ainsi le décalage entre nos perceptions et les perceptions contemporaines pour considérer les auteurs, et leur pensée, au regard de la manière dont ils étaient considérés en leur temps.

10 Le poids des histoires nationales. Depuis la fin du Moyen Âge et le début de l'Époque Moderne, alors même que les cadres nationaux ont conditionné de plus en plus lourdement la pensée juridique, l'Europe n'en est pas moins restée un espace ouvert, permettant aussi bien la circulation des personnes que celle des idées. La prégnance de certains tropismes et de certaines étiquettes nationales ayant longtemps pesé sur l'écriture de l'histoire la pensée juridique, celle-ci doit aujourd'hui se confronter à l'essor de l'histoire transnationale, globale et connectée, comme l'atteste l'essor des travaux portant sur les «transferts culturels » ou la circulation des idées. Si cette perspective pose de nombreux problèmes (J.-L. Halpérin), elle n'en demeure 
pas moins une voie qui, sans être neuve, mérite de nouveaux développements.

11 L'utilité des analyses matérielles et bibliométriques. Encore trop peu pratiquées en histoire du droit, les analyses bibliométriques présentent en matière d'histoire de la pensée juridique de nombreux avantages, qui permettent non seulement de mettre à distance nos connaissances et nos opinions et, par là même, l'historiographie ancienne et contemporaine, mais aussi d'offrir une méthode adaptée aux textes produits par les juristes, ou encore de pouvoir à la fois mettre à l'épreuve nos intuitions, et de générer de nouvelles hypothèses qui parfois ne pourraient voir le jour sans l'outil quantitatif (N. Hakim et A. Monti). Plusieurs articles le démontrent ici grâce à l'emploi du logiciel Gephi(C), lequel utilise des algorithmes construisant des images à partir de données bibliométriques fournies sur les thèmes aussi divers que le secteur du droit des assurances sur la vie, donnant à voir les réseaux entre la France et l'Italie (N. Hakim et A. Monti), la présence des œuvres et des auteurs dans les recueils Sirey et Dalloz (P.-N. Barenot) ou l'étude de la présence de la doctrine juridique française dans le cadre étatsunien (P. Decoux).

La mise à l'épreuve de ces techniques peut certes surprendre, tant par le visuel rendu que par les résultats. Le «smart big data » auquel elle prête vie ne permet pas de faire l'économie d'un travail de fond sur les données recueillies, lesquelles masquent, derrière les chiffres, des réalités potentiellement fort divergentes. L'usage de cette technique permet néanmoins d'affiner considérablement nos connaissances sur la réalité matérielle de la circulation et la réception des œuvres, mais aussi des savoirs et des représentations, sur la mise en réseaux des auteurs, les éventuels phénomènes de transferts, de traductions, d'hybridation, de contamination, d'échanges et de réseaux, aussi bien que de l'historicité de ces circulations, ouvrant de nouveaux horizons pour l'histoire de la pensée juridique.

$$
* * *
$$

13 En amont comme en aval de ce parcours, se trouve posée la question de savoir de quelle pensée juridique nous faisons l'histoire (pour reprendre la formule judicieuse de P.-N. Barenot). L'élargissement des corpus comme celui des méthodes dont l'historiographie contempo- 
raine nous appelle à nous saisir ne peut nous y laisser insensible. En toute hypothèse, cette histoire de la pensée juridique se veut désireuse d'éviter autant que faire se peut d'user de modes de raisonnements et de visions qui sont ceux de nos sociétés contemporaines, mais s'avèrent tout à fait étrangers aux sociétés étudiées, même s'il ne saurait s'agir d'oublier nos présentismes et nos tropismes. Au-delà de ce présupposé épistémologique, la diversité des problématiques envisagées en fonction des périodes, les traitements circonstanciés exigés par l'analyse de sources de nature parfois diamétralement opposées ne saurait permettre d'apporter à la question posée aucune réponse univoque. Pour les uns, doit rester privilégiée une approche de la pensée juridique interne aux juristes, focalisée sur les perspectives unifiantes et les pratiques qui étaient celles, par exemple, des juristes romains, dont l'objectif était précisément la régulation de la vie collective (D. Mantovani). Pour les autres, considérant l'extrême diversité des pratiques d'écritures caractérisant la Renaissance, des perspectives plus larges doivent pouvoir être envisagées, de manière à permettre de sonder les perspectives philosophiques et anthropologiques de ce que l'on considère comme l'avènement de la modernité (G. Cazals). Pour la période contemporaine, en toute hypothèse, il s'agit désormais d'en finir avec l'importance accordée à certaines «cathédrales » doctrinales pour appréhender également une pensée juridique du quotidien, impliquant un droit plus réaliste qu'idéaliste (P.-N. Barenot), de privilégier une « micro-histoire » portant l'individu sur le devant de la scène avec le souci « de rester au plus près du sens porté par le document »(P. Decoux).

14 Ce tableau brossé à grands traits le montre à l'envi : il y a certainement, dans l'histoire de la pensée juridique, des «moments » qu'il appartient de mettre en avant et de sonder (J.-L. Halpérin). Il y a aussi, très clairement, différentes formes de «pensées juridiques » et différentes histoires de la pensée juridique, faisant un usage plus ou moins effectif des différents décloisonnements auxquels appellent les spécialistes contemporains, qu'il s'agisse des champs académiques ou des aires géographiques, notamment, lesquels entravent encore trop souvent la discussion scientifique et le débat historiographique. Pour autant, il y aussi sans nul doute des perspectives et des finalités unitaires qui permettent à ces différentes histoires de se rejoindre : face à la démultiplication à outrance des disciplines et des catégories des 
champs du savoir que voudrait nous imposer une certaine rationalité, l'histoire de la pensée juridique apparaît comme capable de redonner de la lisibilité à une histoire juridique qui reste trop souvent éclatée et tiraillée entre diverses options méthodologiques et politiques. Oui, l'histoire de la pensée juridique est, à n'en pas douter, un sport de combat (pour reprendre l'heureuse expression de C. Jamin).

\section{NOTES}

1 C. Delacroix, F. Dosse, P. Garcia et N. Offenstadt, Historiographies, I : Concepts et débats, «Introduction », Paris, Gallimard, 2010, p. 17.

2 Pour ne citer qu'un exemple, voir C. Delacroix, F. Dosse, P. Garcia, Les courants historiques en France, $\mathrm{xIX}^{e}-\mathrm{xx}^{e}$ siècles, Paris, Armand Colin, éd. 2005.

3 V. par exemple Assaf Likhovski, «The Intellectual History of Law », in Markus Dubber, Christopher Tomlins (eds.), The Oxford Handbook of Historical Legal Research, 2018, forthcoming ; Markus D. Dubber, « Legal history as legal scholarship: doctrinalisme, interdisciplinarity, and critical analysis of law », ibid., forthcoming ; Thomas Duve (ed.), Entanglements in legal history: conceptual approaches, Max Planck Institute for european legal history open access publication, 2014 ; Maksymilian del Mar and Michael Lobban (eds.), Law in Theory and History : new essays on a neglected dialogue, Hart publishing, 2016.

\section{AUTEURS}

\section{Géraldine Cazals}

Université de Rouen, Institut universitaire de France

Nader Hakim

Université de Bordeaux 